\title{
Transparencia en la gestión pública y su incidencia en el derecho al acceso a la información
}

\author{
Transparency in public management and its impact on the right of access to \\ information
}

Transparência na gestão pública e seu impacto no direito de acesso à informação

Vilma Mamani ${ }^{1}$

Universidad Nacional del Altiplano, Perú

Wendy Arisaca

Universidad Andina Néstor Cáceres Velásquez, Perú

DOI: https://doi.org/10.35622/j.rg.2021.03.003

Recibido 30/05/2021/ Aceptado 20/07/2021

\begin{abstract}
RESUMEN. El estudio determinó la transparencia y su incidencia en el acceso a la información pública en la región de Puno. Se diagnosticó el cumplimiento de transparencia en la gestión pública, se describió las condiciones de acceso a la información pública, puesto que el acceso a la información pública es esencial como herramienta de lucha contra la corrupción. Se aplicó el método descriptivo mediante un análisis minucioso que permitió determinar la transparencia también se utilizó la técnica de análisis documental, observación directa. Los resultados indicaron que el 69\% de titulares de las entidades investigadas si cumplieron con la designación del funcionario responsable de la actualización del portal de transparencia y con responsable del acceso a la información pública. El 62\% cumplió con la publicación de los datos generales, siendo obligación del funcionario responsable la actualización de esta información, respecto a la publicación de los instrumentos de gestión. Se puede afirmar que los resultados son óptimos, de este modo los ciudadanos pueden tener acceso a esta información.
\end{abstract}

PALABRAS CLAVE: Acceso a la información, gestión pública, transparencia.

\begin{abstract}
The study determined transparency and its impact on access to public information in the Puno region. Compliance with transparency in general management was diagnosed, the conditions of access to public information were described since access to public information is essential as a tool to fight corruption. The descriptive method was applied through a meticulous analysis that allowed to determine the transparency; documentary analysis and direct observation were also used. The results indicated that $69 \%$ of the heads of the investigated entities did comply with the appointment of the official responsible for updating the transparency portal and responsible for access to public information. Furthermore, $62 \%$ complied with the
\end{abstract}

${ }^{1}$ Correspondencia: Guidojoelmg@gmail.com 
publication of general data, being the responsibility of the responsible official to update this information regarding the publication of management instruments. Therefore, it can be said that the results are optimal; in this way, citizens can have access to this information.

KEYWORDS: Access to information, public management, transparency.

RESUMO. O estudo determinou a transparência e seu impacto no acesso à informação pública na região de puno. Foi diagnosticado o cumprimento da transparência na gestão pública, descritas as condições de acesso à informação pública, uma vez que o acesso à informação pública é essencial como ferramenta de combate à corrupção. O método descritivo foi aplicado por meio de uma análise meticulosa que permitiu determinar a transparência, também foi utilizada a técnica de análise documental, observação direta. Os resultados indicaram que $69 \%$ dos titulares das entidades investigadas cumpriram a nomeação do responsável pela atualização do portal da transparência e responsável pelo acesso à informação pública. 62\% cumpriram a publicação de dados gerais, cabendo ao responsável a atualização desta informação, no que diz respeito à publicação de instrumentos de gestão. Pode-se dizer que os resultados são ótimos, dessa forma os cidadãos podem ter acesso a essas informações.

PALAVRAS CHAVE: Acesso à informação, gestão pública, transparência.

\section{INTRODUCCIÓN}

El derecho de acceso a la información pública es un derecho fundamental, previsto en el Numeral 5 del Artículo $2^{\circ}$ de la Constitución Política del Perú. El derecho al acceso a la información pública data de muchas décadas, gracias a la garantía y reconocimiento universal de los Derechos Humanos. Según Cotino (2017) el principio de máxima transparencia es la base del derecho al acceso a la información, existe algunas restricciones información que califica como secreta, confidencial, reservada contemplada en la norma peruana. Transparencia es uno de los temas más trascendentales, ya que trata de los valores éticos, responsabilidades que todo funcionario realiza durante el ejercicio de sus funciones (Hidalgo, n.d.). En el marco de la administración pública, la transparencia es un mecanismo que previene actos de corrupción y que permite a la ciudadanía conocer el funcionamiento interno de las instituciones y cómo se manejan los fondos que éstas reciben (Romero, 2017) no hay duda de que la apertura de datos públicos incrementa la transparencia sobre las administraciones y sus actuaciones. Por este medio se promueve el ejercicio de la responsabilidad y el buen gobierno, se impulsa el debate ciudadano 
sobre lo público contribuyendo a la lucha contra la corrupción, causante de una creciente desconfianza entre ciudadanos y administraciones (Alonso \& García, 2014).

En la actualidad no existe transparencia absoluta que informe sobre la administración de los recursos públicos generando actos de corrupción, el mal manejo de los fondos públicos en la mayoría de las municipalidades provinciales de la región de Puno (Chambi, 2016), esto es debido a la falta de transparencia de la información pública y la insuficiente capacidad fiscalizadora por parte del estado. Chile es uno de los países latinoamericanos que cuenta con las leyes más completas en transparencia y en el acceso a la información pública, cuenta con una institución autónoma descentralizada encargada de la protección de este derecho (Moreno \& Salazar , 2018).

En el presente estudio se determinó el cumplimiento de transparencia en la gestión pública y las condiciones de acceso a la información en la región Puno,

Para que haya concordancia entre las diferentes disposiciones sobre acceso a la información pública es necesario considerar que el acceso es la información en sí misma que haya sido creada, obtenida o que se encuentre en posesión o bajo el control de la Administración Pública (Quispe, 2016).

\section{MATERIALES Y MÉTODOS}

Para la determinación del cumplimiento de transparencia en la gestión pública en la presente investigación se observaron los portales de transparencia con la finalidad de reunir suficiente información que permita determinar la funcionalidad de los portales, identificar el cumplimiento de cada uno de los rubros detallados en la Directiva $\mathrm{N}^{\circ}$ 001-2017-PCM/SGP.

Metodológicamente, el presente estudio es de tipo descriptivo, puesto que se evaluó el cumplimiento de transparencia y acceso a la información pública en trece municipalidades de la región de Puno determinando si las entidades cumplen con la publicación de información en sus respectivos portales, se determinó si el público 
en general puede tener acceso a la información pública, también se utilizó la técnica de análisis documental esto permitió recopilar información obtenida de libros, informes, tesis, artículos científicos, normas legales, asimismo se aplicó la técnica observación directa, para el procesamiento de la información recopilada se hizo tabulaciones los cuales fueron analizados e interpretados.

\section{Lugar de Estudio}

El área a estudiar son las municipalidades provinciales y distritales de la región de puno de las cuales se seleccionó una muestra de esta forma se analizó la transparencia en la gestión pública. E1 Departamento de Puno está ubicado en la parte sureste del territorio peruano entre los $13^{\circ} 00^{\prime}$ y $17^{\circ} 08^{\prime}$ latitud Sur y en los $71^{\circ} 08^{\prime}$ y $68^{\circ}$ 50' longitud Oeste del meridiano de Greenwich, en un territorio de aproximadamente $72,000 \mathrm{~km}^{2}$, representa el $5.6 \%$ del territorio peruano, con una población de 1'200,000 habitantes, de los cuales el $60 \%$ es rural y el $40 \%$ es urbano.

\section{Tabla 1}

Provincias de la Región Puno

\begin{tabular}{cll}
\hline $\mathbf{N}^{\circ}$ & \multicolumn{1}{c}{ PRoviNCIA } & \multicolumn{1}{c}{ ALCALDE } \\
\hline 1 Municipalidad Provincial de Puno & Ivan Joel Flores Quispe \\
2 Municipalidad Provincial de Azángaro & $\begin{array}{l}\text { Isidro Solorzano Pinaya } \\
\text { Econ. Edwar Rodriguez }\end{array}$ \\
3 Municipalidad Provincial de Carabaya & Mendoza \\
Municipalidad Provincial de Chucuito & \\
4 Juli & Juan CarlosAquino Condori \\
5 Municipalidad Provincial el Collao & Santos Apaza Cardenas \\
6 Municipalidad Provincial de Huancané & Efrain Vilca Callata \\
7 Municipalidad Provincial de Lampa & Clever Huaynacho Hañari \\
8 Municipalidad Provincial de Melgar & Victor Jesús Huallpa Quispe \\
9 Municipalidad Provincial de Moho & Uriel Arcangel Condori Yujra \\
Municipalidad Provincial San Antonio de & \\
10 Putina & Serapio Sucasaire Sucasaire \\
11 & Municipalidad Provincial de San Roman & Oswaldo Marín Quiro
\end{tabular}


12 Municipalidad Provincial de Sandia Miguel Quispe Tipo

13 Municipalidad Provinicial de Yunguyo Juan Huanca Coarita

\section{Descripción detallada por objetivos específicos}

El principio de publicidad garantiza la transparencia en las entidades municipales de la región de Puno, fortaleciendo la vigilancia ciudadana sobre los actos de gestión de la administración pública, es por esto que en el presente trabajo se verificó el cumplimiento de transparencia en la gestión pública.

El acceso a la información pública es relevante pues es una manera que los ciudadanos tienen para controlar la gestión gubernamental, como participar de manera activa con el gobierno y sobre todo evita actos de corrupción por parte de los funcionarios públicos, con lo cual se conocen las condiciones de acceso a la información pública.

\section{RESULTADOS}

\section{Tabla 2}

Designación de funcionario responsable de la Ley de Transparencia y Acceso a la Información Pública

\begin{tabular}{lrrr}
\hline Alternativa & $\begin{array}{c}\text { Frecuencia } \\
\text { Absoluta }\end{array}$ & $\begin{array}{c}\text { Frecuencia } \\
\text { Relativa }\end{array}$ & $\begin{array}{c}\text { Frecuencia } \\
\text { Porcentual }\end{array}$ \\
\hline Designó & 9 & 0.69 & $69 \%$ \\
No designó & 4 & 0.31 & $31 \%$ \\
\hline Total & $\mathbf{1 3}$ & $\mathbf{1}$ & $\mathbf{1 0 0 \%}$
\end{tabular}

Fuente: Portal de Transparencia 


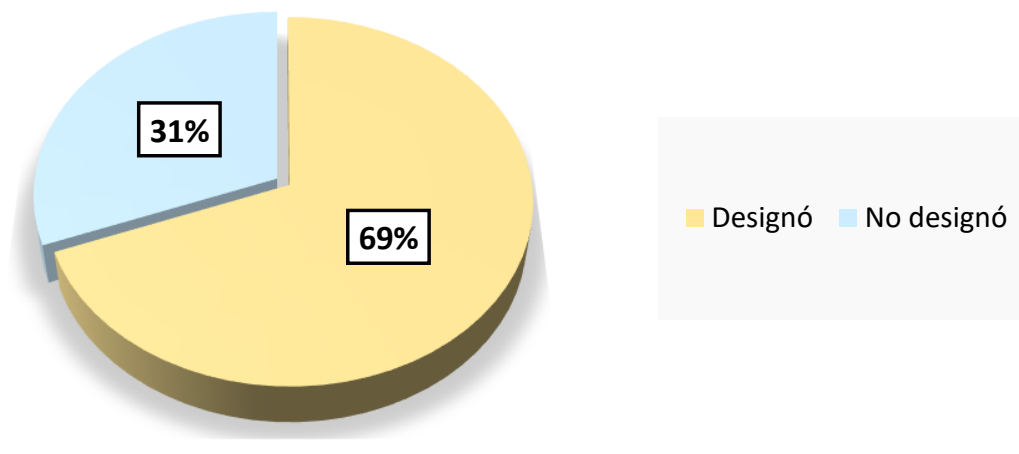

Figura 1 Designación de funcionario responsable de la Ley de Transparencia y Acceso a la Información Pública

Fuente: Tabla 2

Conforme a la Directiva n. ${ }^{\circ}$ 001-2017-PCM/SGP la implementación de esta es obligatoria, el titular de la entidad mediante resolución debe designar; al funcionario responsable de actualizar la información en el portal de transparencia, funcionario responsable de acceso a la información. En la tabla 2 se puede observar que el $69 \%$ de municipalidades cuentan con funcionarios responsables de la implementación de la ley de trasparencia y acceso a la información pública, solo un 31\% de municipalidades no implementaron la Ley, puede existir razones de él porque no implementan, pero están en la obligación de publicar un aviso de sinceramiento dando a conocer los motivos por las cuales no cumplen con la publicación, aun así, las municipalidades no cumplen con tal aviso.

\section{Tabla 3}

Información sobre el cumplimiento de los datos generales en las provincias de la región de Puno

\begin{tabular}{lrrr}
\hline Alternativa & $\begin{array}{c}\text { Frecuencia } \\
\text { Absoluta }\end{array}$ & $\begin{array}{c}\text { Frecuencia } \\
\text { Relativa }\end{array}$ & $\begin{array}{l}\text { Frecuencia } \\
\text { Porcentual }\end{array}$ \\
\hline $\begin{array}{l}\text { Información registrada } \\
\begin{array}{l}\text { Información no } \\
\text { registrada }\end{array}\end{array}$ & 5 & 0.38 & $38 \%$ \\
\hline Total & 8 & 0.62 & $62 \%$ \\
\hline
\end{tabular}

Fuente: Portal de transparencia de las municipalidades 


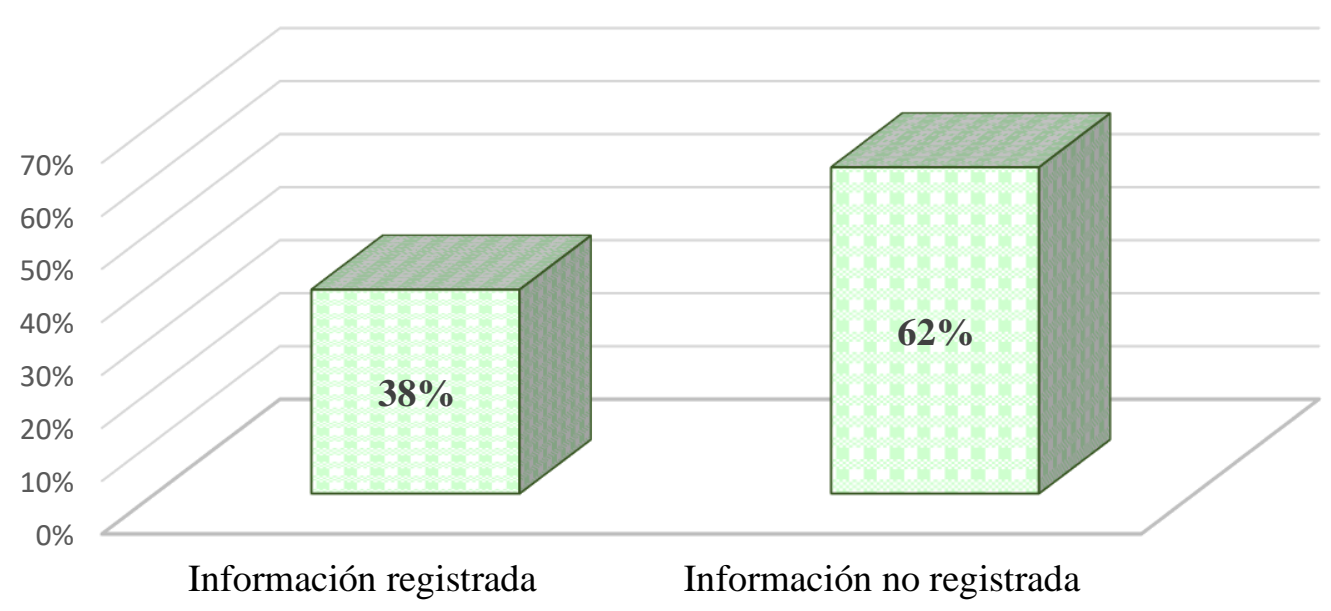

Figura 2 Información sobre el cumplimiento de los datos generales en las provincias de la región de Puno

Fuente: Tabla 3

De la tabla 3 se deduce que el 38\% cumple con la publicidad de información referida a los datos generales que incluye información como el directorio de la entidad, aplicación de normas legales referente a las normas de creación de la entidad, normas emitidas por la entidad resoluciones, ordenanzas, acuerdos entre otros. El $62 \%$ de entidades no cumplen con la publicidad de información es un porcentaje muy alto.

\section{Tabla 4}

La aplicación de instrumentos de gestión en las municipalidades provinciales

\begin{tabular}{lrrrrr} 
Alternativa & ROF & Organigrama & MOF & CAP & TUPA \\
\hline $\begin{array}{l}\text { Información } \\
\text { registrada }\end{array}$ & $77 \%$ & $77 \%$ & $69 \%$ & $69 \%$ & $62 \%$ \\
$\begin{array}{l}\text { Información no } \\
\text { registrada }\end{array}$ & $23 \%$ & $23 \%$ & $31 \%$ & $31 \%$ & $38 \%$ \\
\hline TOTAL & $\mathbf{1}$ & $\mathbf{1}$ & $\mathbf{1}$ & $\mathbf{1}$ & \\
\hline
\end{tabular}

Fuente: Portal de transparencia de las municipalidades 


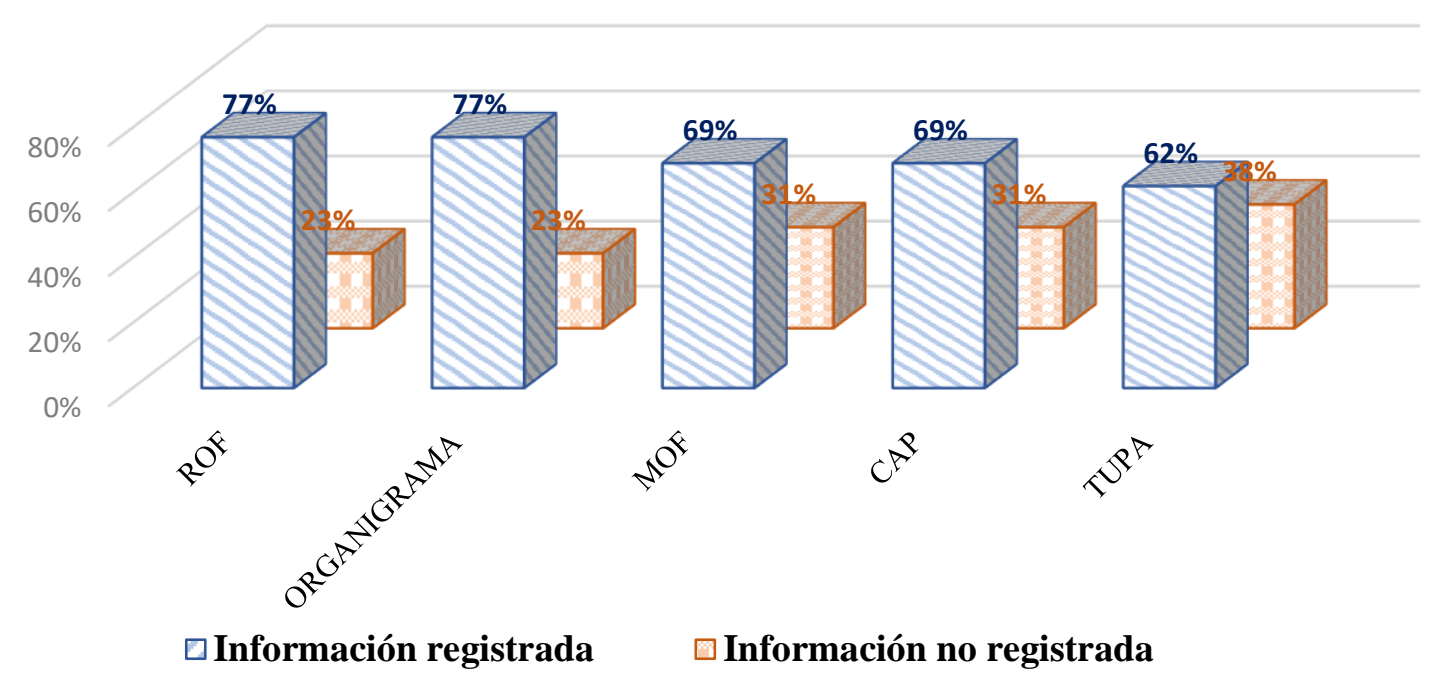

Figura 3 La aplicación de los instrumentos de gestión en las municipalidades provinciales

Fuente: Tabla 4

El acceso a la información es importante las personas tienen derecho a conocer los documentos que producen y administren los servidores públicos, el funcionario responsable de la elaboración y actualización de la información debe publicar en sus respectivos portales los instrumentos de gestión de la investigación realiza se puede deducir que el $77 \%$ cumple con la publicidad de los instrumentos de gestión sean estos Reglamento de Organización y Funciones, Manual de Organización y Funciones, Cuadro de Asignación de Personal, Texto Único de Procedimientos Administrativos entre otros, el 23\% no realizo la difusión de los instrumentos de gestión e incluso algunas entidades no crearon sus portables de transparencia.

\section{Tabla 5}

Publicación de la información presupuestal

\begin{tabular}{|c|c|c|c|}
\hline Alternativa & $\begin{array}{c}\text { Frecuencia } \\
\text { Absoluta }\end{array}$ & $\begin{array}{c}\text { Frecuencia } \\
\text { Relativa }\end{array}$ & $\begin{array}{l}\text { Frecuencia } \\
\text { Porcentual }\end{array}$ \\
\hline Información registrada & 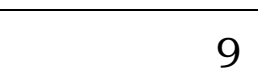 & 0.69 & $69 \%$ \\
\hline Municipalidad Provincial de Puno & & & \\
\hline Municipalidad Provincial de Azángaro & & & \\
\hline Municipalidad Provincial de Carabaya & & & \\
\hline
\end{tabular}


Municipalidad Provincial de Chucuito

Juli

Municipalidad Provincial el Collao

Municipalidad Provincial de Lampa

Municipalidad Provincial de Melgar

Municipalidad Provincial de Moho

Municipalidad Provincial San Antonio

de Putina

Información no registrada

4

0.31

$31 \%$

Municipalidad Provincial de

Huancané

Municipalidad Provincial de San

Román

Municipalidad Provincial de Sandia

Municipalidad Provincial de Yunguyo

\begin{tabular}{llll}
\hline TOTAL & 13 & 1 & $100 \%$ \\
\hline
\end{tabular}

Fuente: Portal de transparencia de las municipalidades

$31 \%$

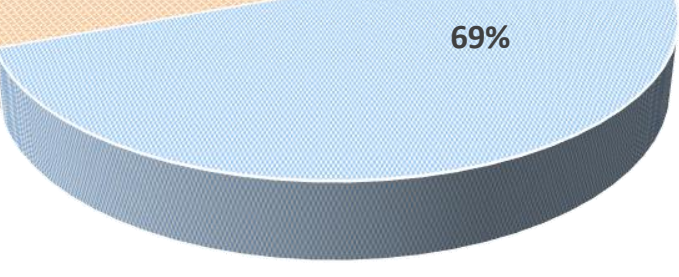

Gráfico 1 Publicación de la información presupuestal

Fuente: Tabla 5

De la tabla 5 se puede afirmar que si cumplen con la publicidad del presupuesto que administran el 69\% de municipalidades si cumplen con la publicidad de esta información esto se debe a que la información que administra el MEF de la ejecución presupuestal se visualiza automáticamente en el portal de transparencia, 31\% no cumple con la publicidad de la información aun cuando existe facilidades de publicidad, pero esto se debe a que estas entidades no realizaron ni la creación de la página web. 


\section{Tabla 6}

Publicación sobre los avances de proyectos de inversión e infobras

\begin{tabular}{ccc}
\hline Alternativa & $\begin{array}{c}\text { Proyectos de } \\
\text { Inversión }\end{array}$ & Infobras \\
\hline Información registrada & $69 \%$ & $62 \%$ \\
Información no registrada & $31 \%$ & $38 \%$ \\
\hline Total & $\mathbf{1}$ & $\mathbf{1}$ \\
\hline
\end{tabular}

Fuente: Portal de transparencia de las municipalidades

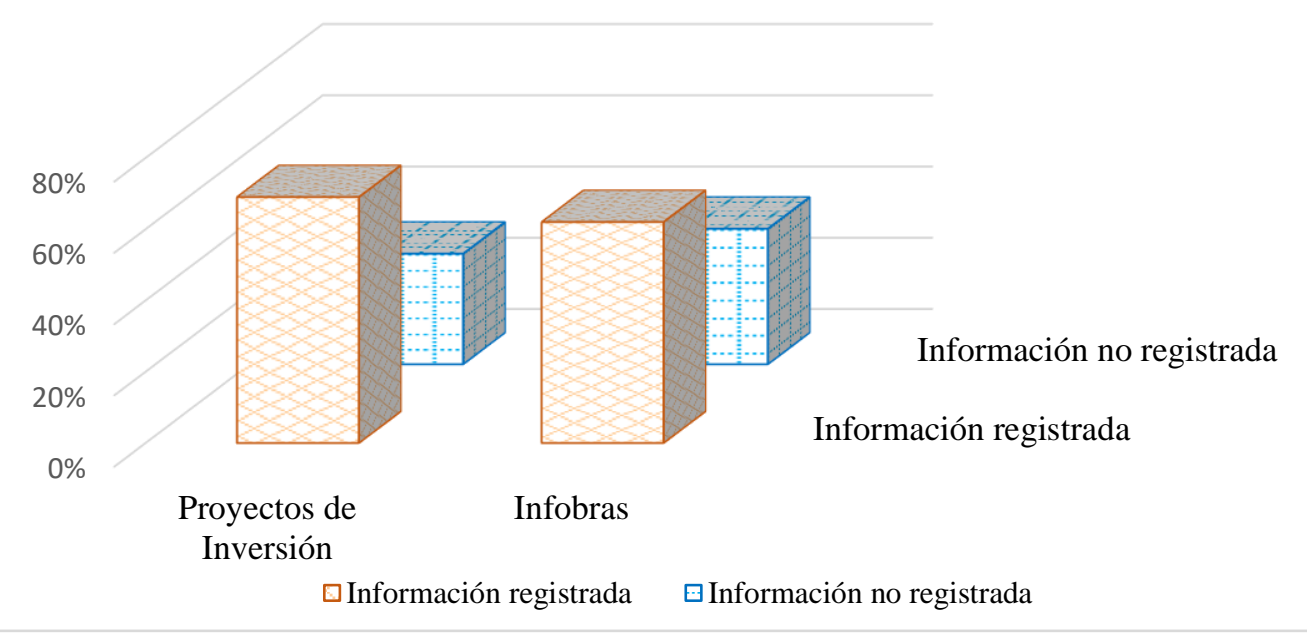

Gráfico 2 Publicación sobre los avances de proyectos de inversión e infobras Fuente: Tabla 6

La información que administra el Sistema Nacional de Inversión Pública que provee el Ministerio de Economía y Finanzas se visualiza automáticamente en el portal de transparencia es por eso que un gran porcentaje de entidades cumplen con tal publicidad, con respecto a Infobras es información que provee la Contraloría General de la Republica y se visualiza automáticamente en el portal.

4. DISCUSIONES 
Conforme con la Resolución Ministerial N035-2017-PCM señala que la implementación del portal de transparencia es de manera obligatoria en las entidades de la Administración Pública para garantizar el principio de publicidad, si bien es cierto el 69\% de titulares de las entidades designó al funcionario responsable para la actualización de los portales de transparencia, pero se puede afirmar que los funcionarios responsables no están cumpliendo de manera correcta con sus funciones.

El acceso a la información pública constituye un derecho fundamental a solicitar y recibir información que poseen las entidades públicas tipificada en el Art. 2 inc. 5 de la Constitución Política del Perú y en el Art.10 de la Ley N²7806 Ley de Transparencia y Acceso a la Información Pública, es importante señalar que todo ciudadano puede ejercer su derecho de acceso a la información pública sea accediendo a los portales de transparencia o mediante una solicitud formal, pero se encuentran con limitaciones para obtener información, ya que los portales de transparencia no se encuentran actualizados.

\section{CONCLUSIONES}

Se ha determinado de una evaluación de 13 portales de transparencia solo nueve cuentan con sus respectivos portales hasta el momento cuentan con la creación de su página web que están en proceso de actualización de información es por ello que es un poco complicado obtener información solo tres municipalidades cumplen con un $70 \%$ de publicidad de información en sus respectivos portales.

Del mismo modo, se determinó $62 \%$ no cumple con la publicidad de datos generales de la entidad como el directorio, normas de creación de la entidad, normas legales, normas emitidas por la entidad.

Se concluye con respecto al rubro de instrumentos de gestión si cumplen con la publicidad de esa información representado un $69 \%$. 


\section{REFERENCIAS}

Alonso Magdaleno, M. L., \& García García, J. (2014). Evaluación de la transparencia municipal en el Principado de Asturia. Auditoría pública: revista de los Organos Autónomos de Control Externo, ISSN 1136-517X, No. 64, 2014, págs. 75-86. Organos autonómicos de control externo. Retrieved from https:/ / dialnet.unirioja.es/servlet/articulo?codigo $=4903557$

Chambi Condori, A. W. (2016). Ausencia de Transparencia en la información pública de los Gobiernos Locales y la Insuficiente Fiscalización del Estado en la Región Puno. Universidad Andina Néstor Cáceres Velásquez. Retrieved from http:// renati.sunedu.gob.pe/handle/sunedu/101892

Cotino Hueso, L. (2017). E1 reconocimiento y contenido internacional del acceso a la información pública como derecho fundamental. Teoria y Realidad Constitucional, ISSN 1139-5583, No 40, 2017, Págs. 279-316, (40), 279-316. Retrieved from https://dialnet.unirioja.es/servlet/articulo?codigo $=6273566$

Hidalgo, C. (n.d.). El proceso de modernización del estado peruano: aspectos importantes a tener en cuenta en la gestión pública. Revistas.Unife.Edu.Pe. Retrieved from http:// revistas.unife.edu.pe/index.php/lumen/article/downloa d/574/489

Moreno, E., Christopher, R., \& Salazar Matias. (2018). Gobernar : the journal of Latin American public policy and governance. Gobernar: The Journal of Latin American Public Policy and Governance, 2(1). Retrieved from https://orb.binghamton.edu/gobernar/vol2/iss $1 / 9$

Quispe, C. (2016). El acceso a datos como parte del derecho de acceso a la información pública. Pontificia Universidad Católica Del Perú. Retrieved from http://tesis.pucp.edu.pe/repositorio/handle/123456789/6704 